\title{
A Critical Outlook on Assumptions- Decision Systems in Transformation Towards Sustainability
}

Some assumptions about decision systems in the context of transformation towards sustainability will be presented in this chapter. These assumptions are backed by rationales, which highlight the utility preferences of agents and audience. In addition, trade-offs reflect the selection of the most important caveats that decision-makers are confronted with. These trade-offs were comprehensively discussed in the previous chapter. Moreover, turnstiles are enumerated that demonstrate possible 'silver-linings' and potential opportunities that may arise to strategically facilitate transformation processes. These turnstiles will be substantially re-examined in a later stage (prescriptive) of this book. The critical assessment of assumptions relevant to the studies of transformation processes towards sustainability needs to be part of any analytical framework. A critical outlook on transformation processes towards sustainability implies a systems analytical approach where the understanding of the parts of human thinking, or elements of reasoning, is given adequate attention. As Richard Paul and Linda Eder (2002) note, an understanding of elements such purpose, question, information, inference, assumption, point of view, concepts, and implications is a prerequisite of any critical thinking. Ignoring unquestioned and blindly accepted assumptions can lead to different types of bias that may have significant impacts, for example, on the relationships between the stakeholders of transformation towards sustainability.

As explained in Chapter 1, the negotiation perspective that defines the analytical framework of this book highlights the justifiability of decisions through the recognition of a diversity of viewpoints. Because most human thinking is inferential in nature (Paul \& Eder 2002), there is a need to uncover, question, clarify and "reconcile" relevant assumptions that guide decisions and reasoning pertaining to dealing with complexity. 'Assumptions' refer to a collection of both justified and 
unjustified beliefs or accurate and inaccurate inferences drawn from experiences, which are then used to interpret the world. Assumptions are those that are taken for granted or presupposed, because they are no longer questioned. Nevertheless, the diversity of inferences is inevitable, because individuals will tend to have different viewpoints or terms of reference on the same situations, which constitute experience and meaning. In other words, because different assumptions can be made about the same situation as reference object, and because assumptions are the basic foundation of decisions and reasoning, the importance of re-visiting assumptions in book needs to be highlighted.

\subsection{Actors}

\section{Assumption 1: Power is to be understood through its 'relative' value.}

Rationale: Excluding some actors to gain access to at least one source of power is impossible, as different types of structural changes unfold, but as different actors express different types of power, there is uncertainty as to which type of power is useful in a specific context or issue. It is important to accept that collective decision-making is a power game that motivates preparations as well as the formulation of short and long-term roadmaps, strategies and contingency plans to facilitate the achievement of goals (see Zartman \& Rubin 2000; Hernandez 2014b). This preparation includes not only the 'calculations' of the 'relative' value of expected outcomes to the status quo to define the legitimacy behind such values, but also the relative value of one's preferred outcome to that of their competitors (Zartman 1994; Dupont \& Faure 2002). In addition, these calculations will usually involve the examination of different types of leverages most likely to be available to oneself and to the others that will define subsequent interactions.

While changing sources of power as well as varying meanings and implications of power asymmetry can mean (old and new) opportunities, this can also be a source of anxieties and insecurities especially under high uncertainty (see Tversky \& Kahneman 1974; Carraro \& Sgobbi 2008). Therefore, it is highly important to assess how sources of power such as access to knowledge, military strength, moral power, and financial capacity can purportedly be converted into political actions by the others. An assessment of power needs must be performed to accommodate the complexity of specific issues. What are the advantages of a country's military strength in achieving climate protection goals?

In addition, the 'relative' value of power can be understood in terms of power asymmetry, whereas the unequal distribution of resources has led to more 
leverage for those with more (see Brooks 2006). It can be assumed that the different pools of actors within the global and national spheres will have different sources and uses of leverage. Nevertheless, having more power can also mean getting less, especially for the one actor with the most power. As power may also mean additional responsibilities and constraints, where the powerful actors are morally obliged to adhere to the rules that they themselves have established. The marginal benefit of one unit of power from the superpower is less than that of a weaker country, particularly because of legitimacy bias. For example, Gilbert Winham and Elizabeth DeBoer-Ashworth (2000) highlight that in the Canada-US Free Trade Agreement between 1985-1987, the more powerful United States was confronted by more urgent issues and so sent less experienced negotiators, while Canada sent its most experienced ones, leading to the best outcome Canada could achieve. Therefore, the concept of rational choice (see Simon 1955; Tversky \& Kahnemann 1986) that assumes 'consistency' among goals and objectives relative to a particular action does not foresee that power asymmetry can mean both advantages and disadvantages. As such, power asymmetry is assumed to prevent many types of consistencies among goals relative to actions. Thomas Hobbes' notion of consistent, value-maximizing reckoning or adaptation within specific constraints (see Friedrich 1963; Gauthier 1969) need to be revisited as actors cannot be consistent with their decisions, as they are compelled to 'compare' values and strategize decisions.

\section{Turnstiles:}

- While the relative valuation of power is captured by its loss-aversion value function, the origins of loss aversion itself can be more effectively identified (and resolved) by framing power within the status quo.

- Power becomes a subject of analysis especially when linked with either maintenance or challenge of the status quo.

- Empathy towards the interests and perspectives of others can become more pronounced after the careful evaluation of relative values.

- Determining relative values can provide more appreciation, as useful insights emerge that can frame debate on values that cannot be measured, such as the value of world heritage sites for humanity.

- Focusing on the relative value of power will most likely allows changes in the distribution of power that may further promote 'decentralization' or 'poly-centralization' of the world order, with more regional powers assuming leadership. 
- The emergence of 'new leaders' or new major players can promote finding 'fresh' ideas and develop innovative leadership styles.

- More deliberative negotiations can be more conducive to robust decisions compared with purely power-driven negotiations, whereas outcomes of deliberative negotiations are more resilient to changes as they are more capable of absorbing shifts in power.

Assumption 2: Power is not only an instrument for achieving goals, it is also a category of identities.

Rationale: As the analysis of power and power asymmetry motivates a more conscious articulation of the relative advantages and disadvantages (see Assumption 1), it can be assumed that behavior reflects purpose or intention. One's purpose or intention is in turn framed by one's expectation of the probability of actually realizing this purpose, which presupposes a calculation of one's power. Furthermore, as the connection between behavior and purpose becomes evident, it can be assumed that decisions (and actions) are framed by a 'value system' as rewards or sanctions are formalized. When an actor follows a value system in its decisions and actions, it is inevitably assuming an 'identity.'

Any form of representation, whether 'formalistic' (see Warren \& Castiglione 2004; Grant \& Keohane 2005) (that is, institutional arrangements that precede and initiate representation), or 'descriptive' (see Pitkin 1967; Young 1986) (that is, the close proximity of the interests between the representative and the represented, or 'symbolic' representation (see Pitkin 1967), presupposes the ability of the representative to project the interests of the represented to the others, as well as a degree of identity-building (see Hernandez 2014a).

In addition, as game theory would suggest, because actor A's choice depends on B's choice (and vice versa) it can also be assumed that the identity of A depends on the identity of B (and vice versa). This mutual causality implies that by making an adjustment to one's decision, an actor can already influence the decisions of the others. This understanding, which conceptualizes the connectivity of decisions, will more likely promote empathy among actors, particularly in the context of power asymmetry.

\section{Turnstiles:}

- As a state or a collectivity assumes an identity, its behavior can become 'predictable' and therefore manageable. Predictability of behavior decreases uncertainty in decision-making processes. 
- Reciprocity can make it easier to forge collaboration and to execute coordination. As parties are able to 'estimate' and 'calculate' the behavior of other actors, they can look for similarities and assess differences. Motivations to agree on concessions increase, because reciprocity enables the creation of additional value.

- Identity-building can promote trust among negotiating actors, primarily because the counterparts are able to identify reference points which allow the others to come up with concrete strategies to respond to or develop a contingency plan to address power asymmetry.

\section{Assumption 3: Rationality is to be decoupled from transitivity.}

Rationale: Decisions are to a significant degree normative and will not always follow transitivity, as rational choice theory assumes. Actors can arbitrarily violate transitivity $(\mathrm{A}>\mathrm{B}>\mathrm{C}$; but $\mathrm{C}>\mathrm{A}$ ) as they assess options (e.g., A, $\mathrm{B}$ or $\mathrm{C}$ ) using their own context-based value systems. Actors may be willing to tolerate the negative aspects of $\mathrm{C}$, although C's pay-offs are worse than those of A. Tolerance is a matter of perception, and emotions can amplify the importance of one or more elements behind an issue while underestimating the others. The consideration of emotions in decision analysis amplifies the human factor and needs to be included in any decision optimization effort (see Moore 1996).

Modern decision theory highlights rational choice in decision-making. Particularly because, in the selection among a set of given options (whose consequences are preferred in terms of the agent's utility function), each set of consequences is ranked according to the order of preference (see Raiffa 2002: 11). However, as suggested by game theory, rational choice can illuminate oversimplifications within the symmetrical normative orientation of actors. In addition, the compulsion to achieve equilibrium frames the set of actions, which highlight the importance of targets (see Raiffa 2002 p. 11). Transitivity is therefore the implication of such oversimplification leading to the failure to grasp complexity.

\section{Turnstiles:}

- By highlighting the 'human factor' (emotions), decision-makers can not only avail themselves of important qualitative impacts, they can also assume a more pragmatic outlook when seeking joint decisions.

- Decoupling transitivity from rationality can allow the development of more useful decision tools that are more effective in managing complexity. 


\section{Assumption 4: Decisions are changed following the adaptation of behaviors through the modification of expected outcomes.}

Rationale: Decisions of actors in a joint decision-making process are made to achieve goals. As goals are converted to outcomes at a later stage, there is a need to conceptualize this 'conversion.' The first step of such a conceptualization is the examination of the 'trigger' that has caused the motivation to formulate goals (see Raiffa 2002 p. 16). The second step involves the format of realizing these goals. Is this format already evident or is there a need to constitute a new one? In cases of problems that can only be solved through cooperation with other actors, there is a need to evaluate how this format can scale up values from interpersonal to larger scales. Assuming the cooperative disposition of human-beings (Messner et al. 2013), it is inevitable that possible motivations for non-cooperation be highlighted. Is this format resulting from group identity formation or is this format an antecedent of such an identity formation? If the latter is the case, there is a need to have a closer look at their existing value system and how their value system dictates specific actions. The next step pertains to the analysis of the meso-level, where persons who represent groups in negotiations affect the course of negotiations, either by promoting or inhibiting the achievement of the goals.

As differences between actors are expected and will be reflected in their behavior when negotiating, the more urgent question is how behavior can be changed while acknowledging these differences. 'Manipulating' behavior refers to altering peoples' choices which will be most likely chosen under business-asusual conditions. Michal Skorepa (2011) suggests that behavior can be manipulated by changing the outcomes of decisions or merely the perceptions about these outcomes. By modifying the volume or context of outcomes, new value is created, paving way for updates in perspectives and behavior. Such a manipulation can be conducted through educational campaigns, where pay-offs can be highlighted or differentiated. Other types of manipulation involve focusing on negative perspectives and on disadvantages (see Ganzach \& Karsahi 1995).

\section{Turnstiles:}

- Modifying outcomes to accommodate changing goals can be done by changing the format of the decision-making process (e.g., from vertical to horizontal)

- Knowing the effectiveness of 'negative framing' in manipulating decisions, decision-makers can assess different types of manipulation, compare these to their own utility functions and 'convert' the negative frame. 
Assumption 5: The compatibility and convertibility of perceptions of justice, fairness and equity define the resilience of decision systems.

Rationale: Sustainable low-carbon transformation encompasses different public goods that are indicated by different connotations and methodologies. As different public good problems (see Snidal 2010) are manifested through the plurality of connotations as well as the relevance of these public goods, several types of strategies emerge. When connotations compete for relevance, decision-making will need to touch on broader principles (environmental protection, civil liberties) and attempt to reconcile those principles in competition.

The existence of differences between actors intending to achieve collective decisions also encompasses differences in perceptions of justice, fairness and equity. The resilience of decision systems is contingent on mechanisms that facilitate the compatibility and convertibility of these differences. While existing orders will most likely reflect an asymmetrical distribution of privileges and (positive and negative) entitlements, the resilience of decision systems, that is, the capacity of these to survive internal and external shocks, will depend on how this asymmetry is reproduced or reinforced by the outcomes. Nevertheless, to understand how the asymmetrical distribution of privileges and entitlements is reproduced or reinforced in outcomes, it is first required to find the typologies of justice, fairness and equity.

\section{Turnstiles:}

- The typologies of justice, fairness and equity as well as the conceptualization of the facilitation of compatibility and convertibility of justice, fairness and equity can be helpful in re-framing conflict cleavages such as the North-South divide, the core-periphery dichotomy, and the urban-rural divide.

Assumption 6: The tolerance (and the acceptability) of biases which are inevitable in the decision-making processes that involve multiple perspectives is highly contingent on mechanisms of persuasion and the possibility of updating perspectives.

Rationale: Often regarded as an 'anomaly', bias is inevitable for any person with a value system. As Johann Galtung (1990) claims, only 'dead people' are free of any form of bias. Human beings, either acting as a person or as a representative of a collectivity, cannot be free from every value system. Such a value system is a collection of life experiences, and although it can be updated, certain elements will remain and will define one's preconception or prejudice. Nevertheless, bias 
does not always need to inhibit decision-making. For example, neutrality or lack of bias is often regarded as a necessary requirement for mediators or chairs in negotiations. However, William Zartman (1988) claims that bias does not disqualify mediators per se, because this bias can be circumvented when mediators who are presumed to be biased are still able to deliver the 'message' from one actor to another. Assuming this logic, bias in collective decision-making processes should be defined as a stumbling block and assessed on how it can inhibit, delay or prevent the realization of presumed goals.

Decision biases are the tendencies to make decisions in certain ways that are systematic deviations from 'rational' or optimal judgment. Psychology and behavioral economics assess these deviations and look for explanations. Biases are often linked to 'heuristics' or 'mental short-cuts' that are cognitively produced to guide decisions. One prominent example of heuristics refers to 'self-fulfilling prophecies' where expectations dictate behavior and positive expectations call for appropriate behavior leading to the realization of goals. On the other hand, negative expectations lead to less motivation and less appropriate behavior, inhibiting the realization of goals. Another example of decision bias pertains to the valuation of 'sunk costs,' also referred to as 'loss aversion.' The pressure of sunk costs, which cause the overvaluing of past investments and the strong determinant of an irrational escalation of commitment (see Staw 1976; Bazerman \& Shonk 2005), may eventually lead to decisions no longer honored, as escalated commitments are simply too expensive. When the disutility of giving up an object is greater than the utility associated with acquiring it (see Kahneman et al. 1991), costs can no longer be legitimized by their objective utility.

Other biases include ambiguity effect or the tendency to avoid options for which missing information makes the probability of an occurrence seem to be unknown (see Baron 1994); anchoring or focalism, that is, the tendency to rely too heavily on one piece of information, usually the first piece of information acquired, when making decisions (see Zhang et al. 2007); availability heuristics or the tendency to overestimate the likelihood of events with greater availability in memory (see Schwarz et al. 1991); the backfire effect, or the reaction to disconfirming evidence by strengthening one's previous beliefs (see Sanna et al. 2002); the bandwagon effect, or the tendency of one's action to occur when many other people are doing the same (see Colman 2003); the bias blind spot, or the tendency to see oneself as less biased than other people or to be able to identify more biases in others than in oneself (see Pronin \& Kugler 2007); the contrast effect, or the enhancement or reduction of a certain perception's stimuli when compared with a recently observed, contrasting object (see Plous 1993); hyperbolic discounting, that is, the tendency for people to have a stronger preference for 
more immediate payoffs relative to later payoffs (see Laibson 1997); and reactive devaluation, or devaluing proposals only because they purportedly originated from an adversary.

\section{Turnstiles:}

- As decision biases are often inevitable, it can be useful to engage a more pragmatic outlook on evaluating biases. One possible step is to make such biases transparent to enable a case-by-case evaluation of their relevance to the realization of goals. As these biases will most likely lead to inconsistent decisions over time, decision-makers will require some mechanisms to document such inconsistent decisions. In addition, what needs to be analyzed is how perspectives can still be updated in spite of the occurrence of biases.

- Personal biases can provide insights into how issues and potential provisions of outcomes are prioritized, thus creating additional contingencies for possible agreements.

- Recognizing bias can allow the justification of compensatory measures, thus the internalization of bias.

\section{Assumption 7: While emotions vindicate the importance of decisions, emo- tions define the unpredictability of decision-making processes.}

Rationale: As the 'human factor' enters the picture, decision-makers are startled by the choices and decisions made by others. Interestingly, human emotions are often linked with irrationality, as emotions hinder humans from achieving the ideal type of homo economicus, which refers to a creature that takes into account all aspects of a situation to maximize its own utility (see Dubreuil 2010). In addition, there is the huge question of how poorly humans are able to relate to the negative emotions of the others, such as anger or fear. Without a sense of empathy, this perplexity leads to frustration. For example, when the lead negotiator of the Philippines delegation broke down in public and wept during the full plenary session of the COP18 climate talks and later staged a hunger strike to force a substantial outcome from the COP meeting, he was celebrated by the media and civil society groups. However, he was also later strongly reprimanded by the Philippine government and was reminded that the COP meeting is not a proper place for such emotional outbursts, as policy-makers need to "show a picture of strength to calm fears" (Cabacungan 2013).

Furthermore, as current developments prove, the rise of social media and other informational and communication technologies have led to the increased claim of 'opinions' as equal to facts and evidence. As such, the transitivity of 
decision-making becomes more difficult, as opinions often refer to an emotional basis. As everyone is deemed to have the right to his or her own opinion, and when policy-making needs to be justified by public opinion, it becomes a question of whether this justification through public opinion another source of unpredictability is, as opinions can quickly change or even be easily manipulated.

The failure to adequately address the gaps between (evidence-based) knowledge systems and self-perceived 'marginalized' individuals has often been addressed by 'correcting the facts.' Nevertheless, as these individuals have crystallized their own identity, stressing the factuality of decisions to 'correct' preconceived and emotion-based ideas and opinions is now counterproductive. In addition, such efforts are resisted as the knowledge system has also been placed into doubt and anti-intellectualism is now equated with 'anti-elitism' or 'anti-establishment' (see Tan 2017).

\section{Turnstiles:}

- New impulses can be found to stress a "we-identity," which can help solve other social cohesion problems.

- The social dimension of policies can gain more attention from policymakers and other stakeholders.

- As knowledge systems are re-visited as a reaction to anti-intellectualism, both methodologies and data assessment systems can be improved and matured.

\subsection{Issues}

Assumption 8: Understanding the domestic (local) in the context of the global and the global in the context of the domestic (local) will provide additional perspectives on the replication of best practices.

Rationale: While states and government agencies have the essential role of conflating global with domestic/local issues, non-government actors have been effective in both 'scaling up' and 'scaling down' good practices. Nevertheless, the replication of best practices, both upwards and downwards, will depend highly on the inclusiveness of decision-making. Increased participation and the enhanced role of particularly marginalized actors need to be dependent not only on the knowledge of what these actors will do, but also on what they could do. Inclusiveness promotes framing and controllability. While multiple perspectives allow issues to be framed differently, they also lead to trade-offs in goals, which highly 
depend on 'costing out' (see Raiffa 2002 p. 19), that is, converting different values to make them relevant, quantifiable, and comparable to other goals.

Inclusiveness also promotes the controllability of the decision-making process, making it more predictable and manageable. A common approach to ensuring inclusiveness is to support NGOs and other social movement organizations, which can serve as bridges between citizens and policy-makers (Princen \& Finger 1994; see Andresen \& Gulbrandsen 2003; Dong Wei 2010). For example, while social movements have developed 'champions' among target groups, they are lobbying for data-driven policies, monitoring and verifying the progress of government policies, seeking partnership or competing with other groups and local communities to advance their goals, and in some cases performing roles traditionally played by governments. Nevertheless, the relationship between governments and social movements is ambivalent and may reflect different developments depending on the local context. While in some countries NGOs have complemented governments in designing and implementing policies, others are viewed as being in 'opposition to the state' particularly when the activities of these NGOs focus on governance issues. In other cases, governments may 'tolerate' NGOs depending on the issues. While the diffusion of power is empowering states against their population, NGOs are also empowered against both their local state and transnationally, against other states, other NGOs and the international society. However, while NGO actions are informed by (liberal) normative concerns, NGOs also pay close attention to instrumental concerns that bear upon organizational survival and growth (Prakash \& Gugerty 2010). Therefore, NGOs and other social movement organizations cannot be exempted from the analysis of power relations.

With the globalization of their means of communication as well as the globalization of their sources of funding, social movements have gained more leverage against government actors. In various cases, social movements have assumed deterrence and balancing strategies, have often been successful in containing state power. As NGOs seek to widen their range of goals and actions in order for them to stay relevant, governments have found ways to embed these groups into the political framework.

\section{Turnstiles:}

- The changing distribution of power between states and NGOs can expand the 'core,' lower barriers for peripheral states to enter the core, and make legitimacy more culturally pluralistic 


\section{Assumption 9: The negative synergies resulting from the interconnectivity of issues will most likely result in an assortment problem.}

Rationale: As a 'portion' or a 'segment' of a (decision) policy is altered to address unacceptable trade-offs or negative externalities to other (decision) policy goals, the overall quality of this initial policy may significantly diminish and/ or may no longer satisfy demand. Therefore, the 'removed' portion or segment needs to be substituted by an adequate component set to avoid 'adaptation loss,' 'linear substitution costs' and 'standardization gaps' (see Sadowski 1959; Pentico 2008). Assortment problems arise when it is difficult to decide which portions or segments of a policy can be altered or even taken out without undermining the overall effectiveness of the policy.

For example, the interconnectivity of promoting renewable energy, local community development, and innovation \& entrepreneurship may result in negative synergies such as the loss of biodiversity which will motivate policy-makers to make changes to their overall sustainable, low-carbon policy to prevent or circumvent the loss of diversity. However, as policy-makers introduce new provisions, identify and implement substitute provisions, or alter existing ones, the effectiveness of the policy may lead to deficiencies.

\section{Turnstiles:}

- The effective management of negative synergies resulting from the interconnectivity of issues can be promoted by a clear understanding of relevant values and pay-offs (what does it bring to the table to address these issues, either only in terms of principles or only their practical meaning).

\subsection{Structures}

Assumption 10: The gaps between individual and collective rationality can be the results of varying levels of decision-making.

Rationale: While unitary decision entities are monolithic and ignore internal conflicts (see Raiffa 2002: 4), collective decisions emphasize the interactions and dynamics between multiple unitary decision entities. Expanding this idea and adopting the process outlook, these interactions and dynamics can be scaled up to various levels or structures. Each scale reflects specific principles, narratives and paradigms. Nevertheless, because these various levels are interconnected, contradiction of principles will only be evident at one point, which will require 
integrated management. In addition, as a joint process is dependent on various factors, both fragmentation and convergence of these levels or structures will most likely be observed, which practically defines institutional complexity.

The implementation of the outcomes of decision-making processes is highly dependent on the connectivity between 'policy' and 'basic games.'

\section{Turnstiles:}

- As different levels of decision-making are highlighted, it can be easier for decision-makers to distribute 'ownership' among them and at the same time forge a shared identity.

- An integrated approach in assessing the various levels (as separate entities) can increase the proximity between policy goals and local conditions leading to enhanced legitimacy and effectiveness.

- The proper analysis of level-dependent rationalities can help develop new tools of evidence-based measurement, data interpretation, monitoring, and assessment.

- Hierarchies place 'anchors' at various stages of decision-making, thus defining expectations and limiting sets of possible outcomes of decision-making processes.

\section{Assumption 11: Decisions cannot be 'freed' from normativity, particularly in the context of transformation processes that involve paradigm shifts.}

Rationale: While the normativity of decisions is well accepted among policy-makers, particularly in the context of climate change mitigation, there has been louder calls for policy-makers to come up with decisions that meet the expectations of the various scientific communities. While these scientific communities, including the IPCC, argue that what they contribute are merely 'policy relevant,' but not 'policy prescriptive,' and when scientific knowledge defines the so-called 'decision maxim,' it inevitably becomes normative, that is, it assumes a prescriptive role. The 'detesting' of the normativity of scientific knowledge follows the self-definition of most scientists of their roles. In order to maintain independence as well as objectivity, scientists need to refrain from any type of normative actions. When politicians, business actors, social groups, etc., are able to dictate the subjects, methods and results of scientific studies, then science loses its integrity.

Nevertheless, particularly when scientific studies define a 'decision maxim,' new discussions are needed on how to address the normative character of these 
studies. A decision maxim is a general maxim (composed of principles and rules), designed such that decision-making may adhere to or violate it (Skorepa 2011: 40). For example, the scientific results synthesized by the various IPCC reports constitute the principles (e.g., $2^{\circ} \mathrm{C}$ threshold) and rules (e.g., life cycle assessment methods) of the decision maxim framing political decisions. Nevertheless, as the next chapter of this book claims, the generation of this knowledge is subject to various norms. Furthermore, should there be consensus about the 'absolute' non-normativity of this scientific knowledge, the 'aesthetic argument,' that according to Michal Skorepa (2011 p. 42) proves the normativity of a decision maxim, shows that the principles and rules also encompass 'aesthetic' elements such as consistency, coherence, stability, symmetry, analogy, balance and simplicity. Scientific knowledge that employs one or more or all of these 'aesthetic' elements is normative per se.

\section{Turnstiles:}

- The acceptance of the normativity of scientific knowledge will most likely require new concepts and methods of accountability and transparency that reveal conflicts of interest.

- The normative outlook on scientific knowledge can be helpful in identifying and 'controlling' various types of decision biases.

\subsection{Processes}

Assumption 12: Global convergence has amplified the 'competition among concepts of modernity' leading to more embedded consolidation of national identities.

Rationale: The increased competition among states implies changes and shifts in the World Order. Nevertheless, the emerging 'Post-Western' World Order will most likely not see the immediate removal of these mechanisms of privilege and vulnerability, but rather maintain them, as these mechanisms are often 'coupled' with rewards and incentives. While some privileges have lost their meaning in terms of 'Realpolitik,' some types of vulnerabilities are either too difficult to eliminate or provide additional political leverage. When this new 'meaning' of vulnerabilities has defined the prospects of alliances, they require a new assessment in terms of international politics. As Barry Buzan and George Lawson (2015) observe, the current World Order is shifting. They claim that the great, old, advanced industrial powers are "exhausted and weakened both materially 
and in terms of ideational legitimacy", debarring them from credibly fulfilling their roles as leaders and giving the impression that the World Order is now defined by the so-called 'G-zero,' which is a World Order where no country can step in to replace the United States. With these powerful countries voluntarily or involuntarily relinquishing their 'exclusive' dominance, more opportunities are given to developing countries with emerging economies to replace these developed countries and set the pace of global decision-making. Nevertheless, these new 'major players' are not always interested to the 'new privileges' linked with global leadership. While some of them have already started establishing and maintaining their 'spheres of influence' (e.g., China in Africa or Southeast Asia), they are resorting to strategies that fall short of global leadership and still demand more responsibilities from the 'old powers', against whom they bring their grievances. The changes and shifts in the World Order have produced a new consciousness and 'naturalness' (Selbstverständlichkeit) that still await conceptualization.

In addition, while the occurring global convergence, as further induced by information technologies, has moved countries closer to each other in terms of economic development, pop culture, Western science (e.g., medicine), democratization, industrialization, and secularization, new waves of 'indigenous modernity' emerge, where various societies recognize that as global convergence unfolds, their national or local identities should be 'empowered.' Various societies that are unable to adapt to new realities without losing their identities are confronted with 'counter-processes' that aim to 'bring back the old glory.' What follows is the 'paradox of modernity', where the struggle between 'competing modernities' (see Mauch \& Patel 2010) has led to more diversity in the definition of modernity. As it is clear that there can be no consensus as to how modernity should be defined, many countries, particularly developing countries, have come up with their own 'indigenous modernity,' which is often seen as challenging, freezing, stagnating or even eroding the world's multilateral system.

\section{Turnstiles:}

- The conflation of global and local cultures will most likely promote co-authorship between the 'old' powers and the 'new' powers in terms of realigning intermediary global and regional institutions, leading to 'global decisions' with more expanded scope and significance.

- A more embedded and consolidated national or local identity will more likely increase the legitimacy of global decisions as it closes the distance between individuals and global decision-makers. 


\section{Assumption 13: Global convergence does not foresee the creation of 'melting pots,' but rather of 'salad bowls'.}

Rationale: The global convergence of standards, norms, governance structures, economic transactions, (popular) culture, consumption, political identities and communication is not contingent upon the creation of 'melting pots,' where differences among actors are 'melted together' to create a homogenous identity. Melting pots address conflict cleavages by eliminating them, whereas alienation among individuals becomes cumulative. Therefore, global convergence needs to be understood as 'salad bowls' where differences mix but still remain in some cases, without causing system ruptures. For example, a melting pot definition of global convergence would see norms spill over to countries which are not always tailored to the local conditions. Melting pots will most likely ignore the relevance of addressing local conditions. In contrast, a salad bowl understanding will see new forms of 'glocalized' decision-making that enjoys more support from local stakeholders (Princen \& Finger 1994; see Roudometof 2015).

Nevertheless, melting pots will exist, but will maintain a rather regional scope, where countries of a specific region benefit from already existing regional cooperation regimes. Regional melting pots will define the thresholds of conflict cleavages, as global transformation will take place not in the global system, but rather in regions. Regional demarcations of knowledge production and representation of the world have defined transfers and synergies within regions. As Barry Buzan and George Lawson (2015) argue, global transformation will be a conglomerate of various (interlinked) transformation processes in various regions, issues and areas.

\section{Turnstiles:}

- New forms of glocalized decision-making will most likely increase the legitimacy of decisions

- Understanding that while problems can be global, their solutions can only be local, will most likely increase ownership of the processes and the solutions, leading to better and more sustainable implementation of goals.

Assumption 14: As decisions relevant to transformation are collective decisions, the achievement of global and domestic/local decisions will depend on how collective and individual 'pay-offs' can be bridged.

Rationale: Negotiations as joint decision-making procedures presuppose that parties voluntarily come together, as the expected 'pay-offs' of individual or unilateral decision-making is 'worse' than those of non-negotiation. Expanding 
upon this, parties will need to 'convert' collective pay-offs in terms of how much these will increase or decrease ones' competitive advantage. This creates the 'negotiation dilemma', where parties are reluctant to commit to negotiated agreements when there is uncertainty about the implications of these agreements in terms of their future negotiation leverage. Therefore, bridging collective and individual pay-offs will also require 'coupling' the costs of non-agreement or non-participation to 'individual loss.' For this to occur, the focus should be shifted from separate interactive actions to group actions, effectively dissolving game theory's 'prisoner's dilemma' (see Raiffa 2002). While parties make separate decisions, these decisions will need to make sense only when they interact with the decisions of others, leading to the production of joint pay-offs.

Nevertheless, joint pay-offs can only be useful when there is full information about individual pay-offs. This will require a specific level of mutual trust, which itself is a subject of learning processes. Joint pay-offs will also depend on reciprocity. On occasions where pay-offs cannot be divided equally, joint pay-offs will be distributed. What follows are exchanges of other resources to 'level up' distribution. Reciprocity plays a role in defining the level of possible trust.

\section{Turnstiles:}

- New methods on analyzing and understanding processes may lead to insights into methods and possibilities of 'coupling' as well as 'decoupling', leading to new values useful for joint pay-offs.

- Bridging collective and individual pay-offs may dissolve free-riding and social traps.

\subsection{Outcomes}

Assumption 15: The efficacy of outcomes of joint decision-making is contingent upon ambiguity that allows constituting exit-strategies from path dependence.

Rationale: While ambiguity may reduce the practical value of outcomes through multiple interpretations of outcomes, it expands the quantity and quality of participation in the decision-making process. Uncertainty around the implementation of outcomes can be relativized when decision pathways, defined as critical junctures, and locked-in sets of decisions, are accompanied by contingencies that allow revisions of path trajectories without forcing a system default or rupture. Decision-making processes that involve more than one actor are particularly 
dependent on provisions that allow flexibility, as there is most likely an asymmetry of departing points. In addition, uncertainty can be managed when decision-making guarantees the repetition of interactions between actors in order to collect experiences and information about the viability of reciprocities. Moreover, because participation in negotiations mutually 'reveals' the identities of participating parties, useful mechanisms of communication can be developed that could, among others, adequately address existing asymmetries.

In the context of negotiations on emission reduction to mitigate climate change, while one actor can easily commit to this mechanism, others will need more time or more capacities, and if not, these actors will not agree on any agreement in the first place. In this situation, flexibility measures entice them to be part of the 'system,' take advantage of the various learning processes and have access to incremental knowledge not only in terms of the technical content of the issues, but also the strength of reciprocities among actors. As such, ambiguity can be regarded as a principle that can initiate (but not guarantee) trust among actors.

From the path dependence perspective (see Arthur 1988; David 1988; Arthur 1994; Sydow et al. 2009), flexibility measures can decrease the 'magnetic' influence of critical junctures and lock-ins. Nevertheless, flexibility measures can be easily 'abused' in order to free-ride. Therefore, the provisions of ambiguity need to be transparent, context-dependent and focused on allowing exit strategies from path dependence. In addition, ambiguity provisions should be limited by complementing them with both quantitative and qualitative indicators of defined targets to promote the predictability of the resulting consequences of 'ambiguous' provisions. Furthermore, the definition of risk profiles (see Raiffa 2002 p. 20) should be clearly communicated. Moreover, the ambiguity of provisions does not always mean uncertainty, as uncertainty pertains to the likelihood of the implementation of the outcome, and ambiguity refers to scalable values such as ordinal ranking, monetary value, desirability value and utility value (see Raiffa 2002: 22).

\section{Turnstiles:}

- Ambiguity provisions for outcomes of joint decision-making can expand participation and deter or even 'convert' spoilers.

- Ambiguity can provide incremental knowledge both in terms of trust and the technical content of relevant issues. 
Assumption 16: Because joint decision-making cannot expect complete information, the implementation of the outcomes of decision-making need to be open to permanent change through learning.

Rationale: In a 'perfect' world, decision-makers will be given a set of alternatives, a set of objectives (or attributes), and a known probability distribution of outcomes and the decision-maker will have a stable utility or preference function (Weber 1987 p. 44). Based on these, an optimal or satisfying solution can be found, whereas the decision-maker is required to provide all information. The decision-maker needs to provide his or her set of objectives to predict his or her behavior. In addition, methods such as conjoint analysis are needed to 'translate' holistic statements into preferences (see Weber 1987). Nevertheless, the 'real world' will be completely different. Not only can all relevant information be known but providing all information that one has can also be practical, particularly in a joint decision-making process involving negotiations. As a bargaining game, negotiators need to calculate how the information he or she gives can lessen his or her leverage. In addition, any prediction about the behavior of an actor based on a set of information can only ever be a model, because humans have both stable and unstable preference structures. Moreover, an incomplete preference structure itself can be the reason for incomplete information, as humans often do not always know what they want (Weber 1987 p. 51). Preferences can also change with or without new information. Therefore, joint decisionmaking should lower its requirement or expectation about the completeness of information.

\section{Turnstiles:}

- Acknowledging the limitations of information can motivate actors to think probabilistically and focus on what they could do and not on what they want to do. In doing so, expectations can be more consistent with the objectives of the decision-making process.

- Although decision-making under incomplete information can promote risk-aversion and increase intractability when achieving outcomes, provisions of permanent change (e.g., conflict management, review and monitoring, etc.) can further induce the learning process. 


\subsection{Interim Conclusion}

This chapter is another important pillar of the theoretical framework. It identified theoretical and conceptual issues in decision systems that tend to be taken as self-evident and therefore ignored, both in theory and in practice. As self-evident assumptions, these issues in decision processes and dynamics tend to be taken for granted. Because of this, the evaluation and the management of these issues are often skipped or circumvented in most functional, institutional and bargaining interactions. They are no longer questioned and therefore they represent missed opportunities. However, they do represent conceptual and practical lock-ins that prevent out-of-the-box or without-a-box thinking that is needed when addressing complex issues of transformation towards sustainability. They can cause further distortions in decision-making. But when they are adequate questions, they might provide additional impulses for strategies and solutions. These issues in decision systems can be understood by exposing them and inviting scholarly discourse on how they can relate to the transformation process towards sustainability.

In this chapter, the exposure of these issues was conducted by explicating them as assumptions and by explaining where these assumptions come from and how these can inhibit or promote transformation towards sustainability. The rationales behind these assumptions intend to shed some light on the utility preferences of agents and audiences. Finally, turnstiles were identified that represent addition opportunities for facilitating the transformation process.

Open Access This chapter is licensed under the terms of the Creative Commons Attribution 4.0 International License (http://creativecommons.org/licenses/by/4.0/), which permits use, sharing, adaptation, distribution and reproduction in any medium or format, as long as you give appropriate credit to the original author(s) and the source, provide a link to the Creative Commons license and indicate if changes were made.

The images or other third party material in this chapter are included in the chapter's Creative Commons license, unless indicated otherwise in a credit line to the material. If material is not included in the chapter's Creative Commons license and your intended use is not permitted by statutory regulation or exceeds the permitted use, you will need to obtain permission directly from the copyright holder.

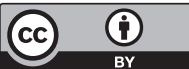

